\title{
Alleviating the Tension in the Cosmic Microwave Background using Planck-Scale Physics
}

\author{
Abhay Ashtekar, ${ }^{1}$ Brajesh Gupt, ${ }^{1}$ Donghui Jeong, ${ }^{2}$ and V. Sreenath ${ }^{3}$ \\ 1 Institute for Gravitation and the Cosmos $\&$ Physics Department, \\ Penn State University, University Park, Pennsylvania 16801, USA \\ ${ }^{2}$ Institute for Gravitation and the Cosmos \& Department of Astronomy and Astrophysics, \\ Penn State University, University Park, Pennsylvania 16801, USA \\ 3 Department of Physics, National Institute of Technology Karnataka, Surathkal, India 575025
}

\begin{abstract}
Certain anomalies in the CMB bring out a tension between the six-parameter flat $\Lambda$ CDM model and the CMB data. We revisit the PLANCK analysis with loop quantum cosmology (LQC) predictions and show that LQC alleviates both the large-scale power anomaly and the tension in the lensing amplitude. These differences arise because, in LQC, the primordial power spectrum is scale dependent for small $k$, with a specific power suppression. We conclude with a prediction of larger optical depth and power suppression in the $B$-mode polarization power spectrum on large scales.
\end{abstract}

Introduction. - The $\Lambda \mathrm{CDM}$ model selected by the PLANCK satellite data has had impressive success in explaining all major features in the temperature anisotropies and polarizations of the cosmic microwave background (CMB), using only six parameters [1]. Let us begin by recalling the procedure that is used to determine the model from the CMB data. Inspired by inflationary models, one assumes that the primordial power spectrum is nearly scale invariant with a specific form, which we will refer to as the standard ansatz (SA):

$$
\mathcal{P}_{\mathcal{R}}(k)=A_{s}\left(\frac{k}{k_{\star}}\right)^{n_{s}-1}
$$

where $A_{s}$ is the amplitude of the scalar mode and $n_{s}$ its spectral index. (Here, $k_{\star}=0.05 \mathrm{Mpc}^{-1}$ is the pivot mode.) To determine a specific $\Lambda \mathrm{CDM}$ model, one requires four additional parameters: $\Omega_{b} h^{2}, \Omega_{c} h^{2}$ that refer, respectively, to baryonic and the cold matter density; and $100 \theta_{*}, \tau$ that determine the observed angular scale associated with acoustic oscillations, and the optical depth that characterizes the reionization epoch 2], respectively. Given the SA and the six parameters, the Boltzmann codes [3 5] that incorporate subsequent astrophysics provides us with four power spectra $C_{\ell}^{T T}, C_{\ell}^{T E}, C_{\ell}^{E E}, C_{\ell}^{\phi \phi}$, where $T, E, \phi$ stand for temperature, $E$-mode (even-parity) polarization, and lensing potential [6, 7]. One compares these theoretical predictions with the observed power spectra and finds the best-fitting values (together with uncertainties) for the six parameters. This fixes the $\Lambda \mathrm{CDM}$ model. One can then work out predictions for other observables, which can be measured independently. For example, the four-point correlation function of the CMB measures the gravitational lensing amplitude $A_{L}$ [8], and the $B$-mode (odd-parity) polarization power spectrum $C_{\ell}^{B B}$ measures the amplitude of tensor perturbation in the early Universe [9, 10].

At the same time, the CMB data exhibit some anomalies that bring out tensions between the best-fit $\Lambda \mathrm{CDM}$ model and observation. We will ignore the tension between the CMB and low- $z$ observations, and focus instead on two anomalies in the CMB. The first is the large-scale power anomaly related to $S_{1 / 2} \equiv \int_{-1}^{1 / 2}[C(\theta)]^{2} d(\cos \theta)$, obtained by integrating the two-point correlation function $C(\theta)$ of the CMB temperature anisotropies over large angular scales $\left(\theta>60^{\circ}\right)$. The WMAP [11, 12] and PLANCK 13, 14] measured values of $S_{1 / 2}$ are much smaller than the expectation from the SA $+\Lambda \mathrm{CDM}$ cosmology. The second is the anomaly associated with the lensing amplitude $A_{L}$. When it is allowed to vary, $A_{L}$ prefers a value larger than unity, hinting at an internal inconsistency in the $\Lambda \mathrm{CDM}$ cosmology 7, 15 19 based on the SA. In particular, it was recently suggested [20] that this anomaly gives rise to a "possible crisis in cosmology" because the positive spatial curvature one can introduce to alleviate this tension makes CMB analysis inconsistent with low- $z$ cosmological measurements.

In this Letter, we present an intriguing possibility of alleviating both anomalies within a well-motivated theoretical framework of loop quantum cosmology (LQC). First, the LQC prediction modifies the SA for the primordial power spectrum by suppressing its large-scale amplitude, which naturally leads to lower $S_{1 / 2}$. The scaledependent primordial power spectrum, in turn, prefers a higher amplitude $A_{s}$ that pushes lensing amplitude $A_{L}$ toward unity (making it consistent with flat $\Lambda \mathrm{CDM}$ ), and higher optical depth $\tau$. Finally we show that, due to the modified primordial power spectrum and higher $\tau$, LQC leaves a specific signature in the $B$-mode (odd-parity) polarization power spectrum.

Modified primordial power spectrum. - In LQC, the big bang singularity is naturally resolved and replaced by a big bounce (see, e.g., Refs. [21, 22]). Therefore, one can systematically investigate the dynamics of cosmological perturbations in the pre-inflationary epoch starting from the Planck regime (see, e.g., Refs. [23 34]). Since the quantum corrected Einstein's equations never break down, all physical quantities remain finite. In particular, while the scalar curvature $R$ of space-time diverges at the big bang, it remains finite at the bounce, achiev- 
ing its universal maximum value $R_{\max } \approx 62$ in Planck units. Now, curvature - more precisely $R / 6$ - provides a natural scale in the dynamics of the gauge invariant perturbations (which in de Sitter space-time coincides with $\left.2 H^{2}\right)$. Fourier modes with physical wave numbers $k_{\text {phys }} \equiv k / a(\eta) \gg(R / 6)^{1 / 2}$ are essentially unaffected by curvature while those with $k_{\text {phys }} \lesssim(R / 6)^{1 / 2}$ get excited. Therefore the evolution during the preinflationary epoch of LQC is subject to a new scale: $k_{\mathrm{LQC}}=\left(R_{\max } / 6\right)^{1 / 2} \approx 3.21$ in Planck units. Modes with $k_{\text {phys }}^{\mathrm{B}} \lesssim k_{\mathrm{LQC}}$ at the bounce are excited during their preinflationary evolution. Therefore they are not in the Bunch Davies (BD) vacuum at the onset of the relevant slow roll phase of inflation-i.e., a couple of $e$-folds before the time at which the mode with the largest observable wavelength crosses the Hubble horizon during inflation. (For details, see Refs. 23, 24]).

Now, one's first reaction may be that these excitations are observationally irrelevant because they would be simply diluted away by the end of inflation. However, this is not the case: because of stimulated emission, the number density of these excitations remains constant during inflation [22, 35, 36]. Therefore the primordial LQC power spectrum at the end of inflation is different from the standard ansatz of Eq. (1) for modes with $k_{\text {phys }}^{\mathrm{B}}<k_{\mathrm{LQC}}$.

The key question then is whether these long wavelength modes are in the observable range. The answer depends on the choice of the background metric that satisfies the quantum corrected Einstein's equations of LQC, and the Heisenberg state of the cosmological perturbations. In standard inflation, the background metric can be any solution of Einstein's equation for the given potential, and, since one cannot specify the quantum state of perturbations at the big bang, one specifies it, so to say, in the middle of the evolution by asking that they be in the $\mathrm{BD}$ vacuum at the start of the relevant phase of the slow roll. In LQC, geometry is regular at the bounce. Using this fact, key features of the quantum geometry in LQC, and a "quantum generalization" of Penrose's Weyl curvature hypothesis [37], a specific proposal has been put forward to make the required choices [30, 31]. Quantum corrected LQC dynamics then leads to unique predictions for the primordial power spectrum for any given inflationary potential; there are no parameters to adjust. The viewpoint is to use the proposal as a working hypothesis, analyze the consequences, and use the $\mathrm{CMB}$ observations to test its admissibility.

The proposal constrains the background metric to be such that the $\Lambda \mathrm{CDM}$ universe has undergone $\simeq 141 \mathrm{e}$ folds since the bounce (irrespective of the choice of inflationary potential) 30]. It then follows that the mode with $k_{\text {phys }}=k_{\mathrm{LQC}}$ at the bounce has comoving wave number $k_{\circ} \simeq 3.6 \times 10^{-4} \mathrm{Mpc}^{-1}$. The primordial power spectrum of LQC is nearly scale invariant for $k \gg k_{\circ}$ but power is suppressed for $k \lesssim 10 k_{\circ}$ :

$$
\mathcal{P}_{\mathcal{R}}^{L Q C}=f(k) A_{s}\left(\frac{k}{k_{\star}}\right)^{n_{s}-1}
$$

where the form of the suppression factor $f(k)$ can be seen in Fig $1\left[f(k) \approx 1\right.$ for $k \gg k_{\circ}$.] This difference from the standard ansatz can be traced back directly to the modes not being in the BD vacuum at the onset of inflation. Now, if the total energy in the scalar field is dominated by the kinetic contribution at the bounce, details of the potential do not affect the preinflationary dynamics, and the suppression factor $f(k)$ is also the same. Analysis of Ref. [38] strongly suggests that there is a large class of potentials for which our proposal to choose the background geometry will constrain the bounce to be kinetic energy dominated. This is illustrated by comparing the Starobinsky inflation 39] and the quadratic potential in Fig!1.

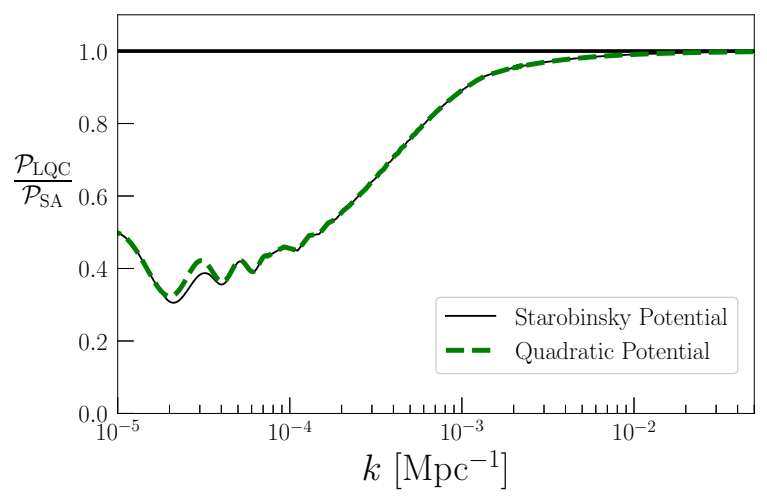

FIG. 1: Ratio of the primordial scalar-power spectrum for LQC and SA. Power is suppressed in LQC for $k \lesssim 10 k_{\circ} \simeq$ $3.6 \times 10^{-3} \mathrm{Mpc}^{-1}$. Plots for the Starobinsky and quadratic potentials are essentially indistinguishable.

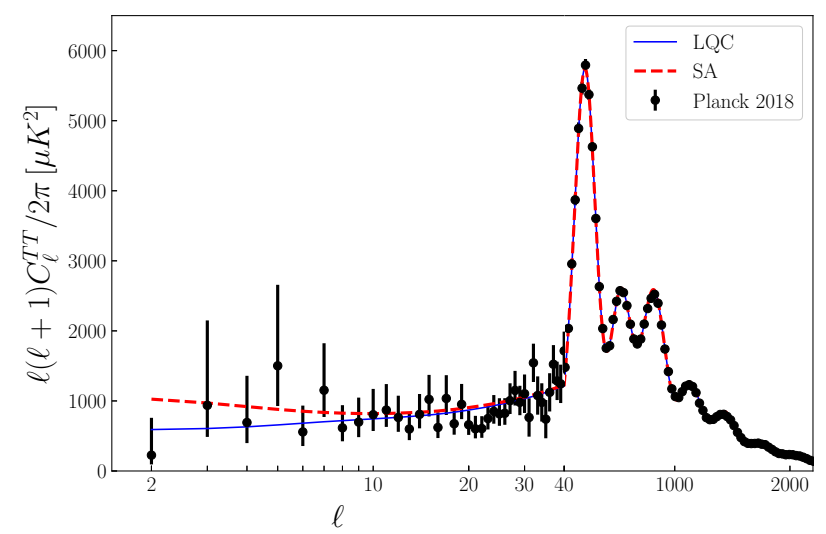

FIG. 2: TT power spectra. The 2018 PLANCK spectrum (black dots with error bars), the LQC [solid (blue) line], and the standard ansatz predictions [dashed (red) line].

Results.-All results are based on the PLANCK-2018 data [1] using the observed TT, TE, EE, and $\phi-\phi$ power 
spectra (including the $\ell<30$ modes for EE correlations) to which the associated likelihoods are Planck TTTEEE+lowl+lowE+Lensing.

Figure 2 shows the observed TT-power spectrum together with the $1 \sigma$ (68\% confidence level) error bars, and the LQC and the SA predictions for the respective bestfit cosmological parameters. Clearly, LQC power is suppressed at $\ell \lesssim 30$ relative to the $\mathrm{SA}$. This is also true for the EE power spectrum (as already noted in Ref. [30], using the then available PLANCK 2015 data). Note that the difference between LQC and SA best-fitting curves shown in Fig. 2 underestimates the difference in the predicted primordial spectra, for the best-fitting cosmological parameters are different. Also, had the LQC $+\Lambda \mathrm{CDM}$ model been used for their analysis, the cosmic-variance uncertainties on large scales may have been smaller than the reported values from PLANCK 2018.

Figure 3 compares the angular TT two-point correlation function $C(\theta)$ predicted by $\mathrm{LQC}$ with that predicted by the SA. It is clear by inspection that the LQC prediction for $C(\theta)$ is closer to the observed values for all $\theta$. In order to quantify this difference, we computed $S_{1 / 2}$. As the last row of Table I shows, the $S_{1 / 2}$ from the best-fit LQC $+\Lambda \mathrm{CDM}$ model is about a third of that obtained from $\mathrm{SA}+\Lambda \mathrm{CDM}$, and closer to the value of $S_{1 / 2}=1209.2$ given by the PLANCK Collaboration using the Commander CMB map. But since that value is obtained after masking and additional processing, a more appropriate comparison would be with the value 6771.7 of $S_{1 / 2}$ obtained from the full sky map, i.e. using the PLANCK $C^{T T}$ data for all $\ell$. The difference between LQC and this PLANCK value is also significantly lower than that between SA and this PLANCK value. This is a substantial alleviation of the tension between theory and observations that has been emphasized over the years [11 14].

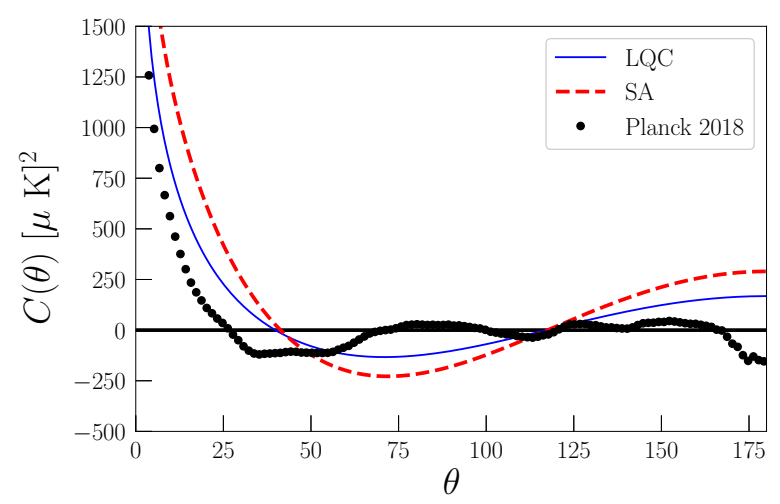

FIG. 3: The angular power spectrum $C(\theta)$. The 2018 PLANCK spectrum (thick black dots), the LQC [solid (blue) line], and the standard ansatz [dashed (red) line] predictions. Values of cosmological parameters are fixed to the mean values given in Table

\begin{tabular}{|c|c|c|}
\hline Parameter & SA & LQC \\
\hline \hline$\Omega_{b} h^{2}$ & $0.02238 \pm 0.00014$ & $0.02239 \pm 0.00015$ \\
\hline$\Omega_{c} h^{2}$ & $0.1200 \pm 0.0012$ & $0.1200 \pm 0.0012$ \\
\hline $100 \theta_{M C}$ & $1.04091 \pm 0.00031$ & $1.04093 \pm 0.00031$ \\
\hline$\tau$ & $0.0542 \pm 0.0074$ & $0.0595 \pm 0.0079$ \\
\hline $\ln \left(10^{10} A_{s}\right)$ & $3.044 \pm 0.014$ & $3.054 \pm 0.015$ \\
\hline$n_{s}$ & $0.9651 \pm 0.0041$ & $0.9643 \pm 0.0042$ \\
\hline \hline$S_{1 / 2}$ & 42496.5 & 14308.05 \\
\hline
\end{tabular}

TABLE I: Comparison between the standard ansatz and LQC. The mean values of the marginalized PDF for the six cosmological parameters, and values of $S_{1 / 2}$ calculated using $C_{\ell}^{\mathrm{TT}}$.

Table I also shows the mean values of the marginalized probability distributions of the six cosmological parameters together with their $1 \sigma$ ranges. For the first three, namely, $\Omega_{b} h^{2}, \Omega_{c} h^{2}$, and $100 \theta_{M C}$, the difference between the $\mathrm{SA}+\Lambda \mathrm{CDM}$ and $\mathrm{LQC}+\Lambda \mathrm{CDM}$ values is $<0.07 \sigma$ and for $n_{s}$ the difference is $\sim 0.2 \sigma$. However, the values of the optical depth $\tau$ and $\ln \left(10^{10} A_{s}\right)$ have increased in LQC by $0.72 \sigma$. As we discuss below, this significant change is a direct consequence of the scale-dependent initial power spectrum (2) of LQC, which also leads to a $0.56 \sigma$ decrease in the lensing amplitude $A_{L}$ from $1.072 \pm 0.041$ in $\mathrm{SA}+\Lambda \mathrm{CDM}$ to $1.049 \pm 0.040$ in $\mathrm{LQC}+\Lambda \mathrm{CDM}$, when $A_{L}$ is also varied. Furthermore, when $A_{L}$ is included in the analysis, the $\Lambda \mathrm{CDM}$ parameters change by $0.59 \sigma-1.48 \sigma$ in SA and $0.39 \sigma-1 \sigma$ in LQC. As Fig. 4 shows, the value $A_{L}=1$ lies outside of the $68 \%$ confidence level for the $\mathrm{SA}+\Lambda \mathrm{CDM}$ model (red contours). A natural way to alleviate this tension within the $\mathrm{SA}+\Lambda \mathrm{CDM}$ is to consider a closed universe. However, then other disagreements with observations arise that prompted the authors of Ref. [20] to raise the possibility of a "crisis in cosmology." What is the situation with the altered values of $\tau$ and $A_{L}$ in LQC? We see from Fig. 4 that now the tension is naturally alleviated because the value $A_{L}=1$ is within $68 \%$ confidence level (blue contours). Therefore, the primary motivation for introducing spatial curvature no longer exists in LQC.

General implications of power suppression at large angles. - In LQC, the mechanism for departure from the nearly scale invariant ansatz (1) is rooted in fundamental considerations in the Planck regime. Nonetheless, it is natural to ask if the qualitative features of some of our results will carry over if there were other mechanisms that led to the primordial spectrum of the form given in Eq. (2). We now show that this is indeed the case.

Let us then suppose that there is some mechanism that provides a primordial power spectrum of the form (2) for some $k_{\circ}$. Let us compare and contrast the resulting best fit $\Lambda$ CDM model with that given by the SA of Eq. (11). As a first step, let us restrict the analysis only to smaller 


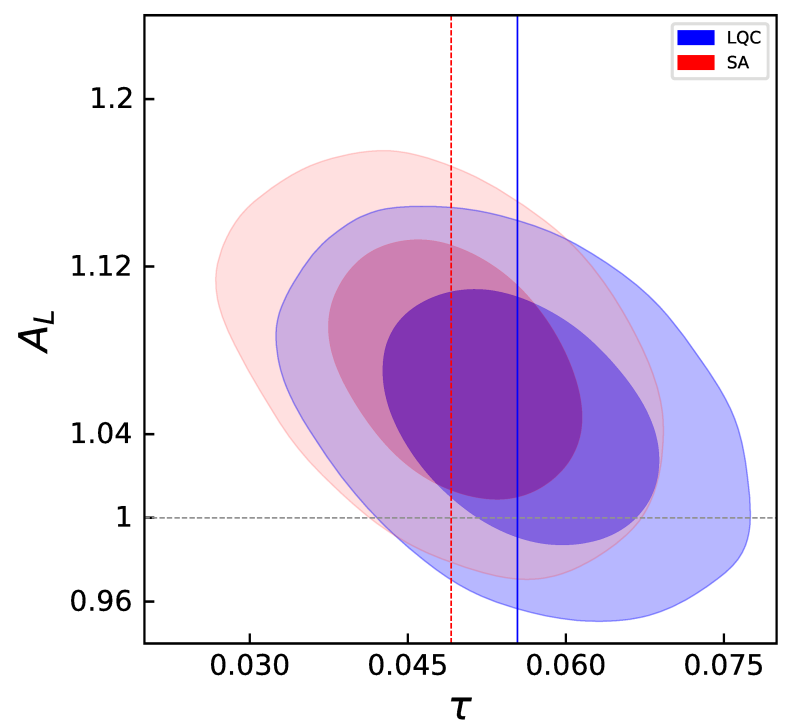

FIG. 4 : $1 \sigma$ and $2 \sigma$ probability distributions in the $\tau-A_{L}$ plane. Predictions of the standard ansatz (shown in red) and LQC (shown in blue). Vertical lines represent the respective mean values of $\tau$.

angular scales $\left(k \gg k_{\circ}\right)$. Then, the primordial spectrum in both schemes is the same, whence we will obtain the same best fit values of the six cosmological parameters. Denote by $\AA_{s}$ the best fit value of the scalar amplitude $A_{s}$. In the second step, let us bring in the full range of observable modes including $k \leq k_{\circ}$. Now, given the $o b$ served large-scale suppression in the TT power spectrum, for $\mathrm{SA}+\Lambda \mathrm{CDM}$ model the best-fit value $A_{s}^{(1)}$ for the entire $k$ range will be lower than $\AA_{s}$. By contrast if the primordial power spectrum is of the form of Eq. (2), $\AA_{s}$ will not have to be lowered as much to obtain the best fit $A_{s}^{(2)}$ since the initial power is already suppressed by $f(k)$. Thus, we have $\AA_{s}>A_{s}^{(2)}>A_{s}^{(1)}$. [For the $f(k)$ in LQC, we have $\ln \left(10^{10} \AA_{s}\right)=3.089$ and $\ln \left(10^{10} A s^{(2)}\right)=3.054$ and $\ln \left(10^{10} A s^{(1)}\right)=3.044$.] The key point is the last inequality: $A_{s}^{(2)}>A_{s}^{(1)}$. Now, we know that for large $k$, the product $A_{s} e^{-2 \tau}$ is fixed by observations. Hence, it follows that the best fit values of the optical depth in the two scheme must satisfy $\tau^{(2)}>\tau^{(1)}$. Finally, from the very definition of lensing amplitude, the value of $A_{L}$ is anticorrelated to the value of $A_{s}$. Therefore, we will have $A_{L}^{(2)}<A_{L}^{(1)}$. Thus in any theory that has primordial spectrum of the form (2), $A_{s}, \tau$, and $A_{L}$ will have the same qualitative behavior as in LQC, and hence the tension with observations would be reduced. What LQC provides is a precise form of the suppression factor $f(k)$ from "first principles," and hence specific quantitative predictions. The LQC $f(k)$ also leads to other predictions - e.g., for the $\mathrm{BB}$ power spectrum discussed below - that need not be shared by other mechanisms.

Summary and discussion.-In LQC, curvature never diverges and reaches its maximum value at the bounce. As a result, preinflationary dynamics naturally inherits a new scale, $k_{\mathrm{LQC}}$, such that modes with $k_{\text {phys }}^{B} \lesssim k_{\mathrm{LQC}}$ at the bounce are not in the BD vacuum at the start of the slow roll phase of inflation [23, 24], whence the primordial power spectrum is no longer nearly scale invariant, but of the form (2). The LQC dynamics and initial conditions then imply 30] that there is power suppression in $\mathrm{CMB}$ at the largest angular scales $\ell \lesssim 30$. In contrast to other mechanisms that have been proposed, this suppression has origin in fundamental, Planck scale physics rather than in phenomenological adjustments put in by hand just before or during the slow roll. As a result of this power suppression, there is an enhancement of optical depth $\tau$ and suppression of the lensing potential $A_{L}$. The two together bring the value $A_{L}=1$ within $1 \sigma$ of the LQC $\tau-A_{L}$ probability distribution, thereby removing the primary motivation for considering closed universe and the subsequent "potential crisis" 20]. In addition, the anomaly in $C(\theta)$ at large angles $11-14$ is significantly reduced; the LQC value of $S_{1 / 2}$ is $\sim 0.34$ of that predicted by standard inflation. The PLANCK Collaboration had suggested [1] that "... if any of the anomalies have primordial origin, then their large scale nature would suggest an explanation rooted in fundamental physics. Thus it is worth exploring any models that might explain an anomaly (even better, multiple anomalies) naturally, or with very few parameters." In this Letter we presented a concrete realization of this idea. (For an alternate proposal within LQC see Ref. [40]).

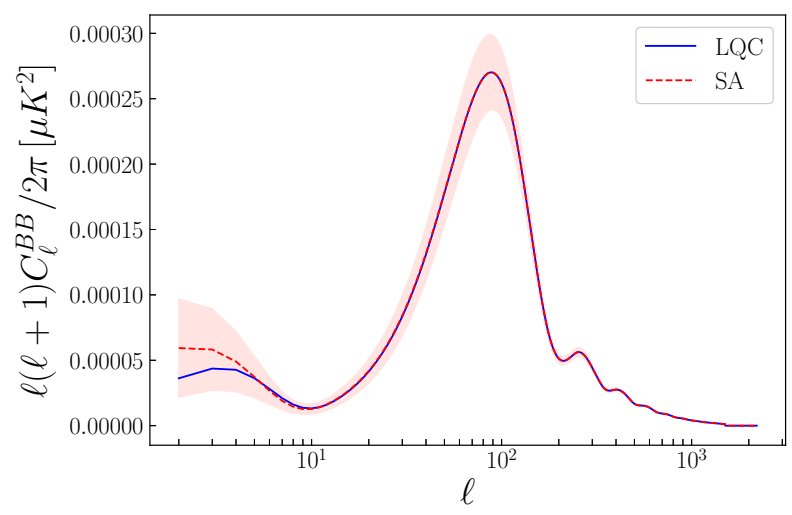

FIG. 5: Predicted Power spectra for BB polarization with $1 \sigma$ uncertainty. Comparison between LQC and standard inflation. The tensor to scalar ratio $r$ has been set to 0.0041 , motivated by Starobinsky inflation [39]. The shaded region indicates the cosmic variance for SA.

This model also leads to other specific predictions. First, as Table I shows, the reionization optical depth $\tau$ is predicted to be $\sim 9.8 \%$ (i.e., $0.72 \sigma$ ) higher. This prediction can be tested by the future observation of global 21 $\mathrm{cm}$ evolution at high redshifts that can reach a percent level accuracy in the measurement of $\tau$ [41]. Second, for 
any given inflationary potential, the primordial spectra of LQC and SA share the same value of $r$ - the tensor to scalar ratio - which depends on the potential. But there is a specific scale dependence in the large-scale $B$-mode (odd-parity) polarization power spectrum, as shown in Fig. 5. The difference is driven by the LQC suppression of the primordial tensor amplitude combined with the larger reionization contribution due to higher $\tau$. Provided that $r$ is sufficiently large, for example, $r \gtrsim 0.001$, we may be able to test this prediction against the data from the future $B$-mode missions such as LiteBIRD [42], Cosmic Origins Explorer [43], or Probe Inflation and Cosmic Origins (PICO [44]). Again, LQC modifies $C_{\ell}^{B B}$ on large scales where the cosmic variance limits its detectability. However, in light of results presented in this Letter, we hope that the LQC primordial power spectrum will be included in the future cosmological analysis.

We would like to thank Ivan Agullo and Charles Lawrence for valuable inputs and Pawel Bielewicz for help with Fig. 3. This work was supported in part by the NSF Grants No. PHY-1505411 and No. PHY1806356, the NASA ATP Grant No. 80NSSC18K1103, and the Eberly research funds of Penn State. V.S. was supported by the Inter-University Centre for Astronomy and Astrophysics, Pune during the early stages of this work. Portions of this research was conducted with high performance computing resources provided by Louisiana State University [45].

[1] Y. Akrami et al. [Planck Collaboration], "Planck 2018 results. I. Overview and the cosmological legacy of Planck", arXiv:1807.06205 [astro-ph.CO].

[2] R. Adam et al. [Planck Collaboration], "Planck intermediate results. XLVII. Planck constraints on reionization history," Astronomy \& Astrophysics. 596, A108 (2016).

[3] U. Seljak and M. Zaldarriaga, "A Line of sight integration approach to cosmic microwave background anisotropies," Astrophys. J. 469, 437 (1996), astro-ph/9603033.

[4] A. Lewis, A. Challinor and A. Lasenby, "Efficient computation of CMB anisotropies in closed FRW models," Astrophys. J. 538, 473 (2000), astro-ph/9911177;

[5] D. Blas, J. Lesgourgues and T. Tram, "The Cosmic Linear Anisotropy Solving System (CLASS) II: Approximation schemes," JCAP 1107 (2011) 034, arXiv:1104.2933 [astro-ph.CO]].

[6] N. Aghanim et al. [Planck Collaboration], "Planck 2018 results. V. CMB power spectra and likelihoods," arXiv:1907.12875 [astro-ph.CO].

[7] N. Aghanim et al. [Planck Collaboration], "Planck 2018 results. VI. Cosmological parameters," arXiv:1807.06209 [astro-ph.CO]].

[8] W. Hu and T. Okamoto, "Mass reconstruction with cmb polarization," Astrophys. J. 574, 566 (2002), astro-ph/0111606.

[9] M. Kamionkowski, A. Kosowsky and A. Stebbins, "Statistics of cosmic microwave background polariza- tion," Phys. Rev. D 55, 7368 (1997), astro-ph/9611125.

[10] U. Seljak and M. Zaldarriaga, "Signature of gravity waves in polarization of the microwave background," Phys. Rev. Lett. 78, 2054 (1997), astro-ph/9609169.

[11] D. N. Spergel et al. [WMAP Collaboration], "First year Wilkinson Microwave Anisotropy Probe (WMAP) observations: Determination of cosmological parameters," Astrophys. J. Suppl. 148, 175 (2003), astro-ph/0302209.

[12] D. Sarkar, D. Huterer, C. J. Copi, G. D. Starkman and D. J. Schwarz, "Missing power vs low-l alignments in the cosmic microwave background: No correlation in the standard cosmological model," Astropart. Phys. 34, 591 (2011), arXiv:1004.3784 [astro-ph.CO]].

[13] Y. Akrami et al. [Planck Collaboration], "Planck 2018 results. VII. Isotropy and statistics of the CMB," arXiv:1906.02552 [astro-ph.CO].

[14] D. J. Schwarz, C. J. Copi, D. Huterer and G. D. Starkman, "CMB anomalies after Planck," Class. Quant. Grav. 33, no. 18, 184001 (2016), arXiv:1510.07929 [astro-ph.CO]].

[15] P. Motloch and W. Hu, "Tensions between direct measurements of the lens power spectrum from Planck data," Phys. Rev. D 97, no. 10, 103536 (2018), arXiv:1803.11526 [astro-ph.CO]].

[16] F. Couchot, S. Henrot-Versill, O. Perdereau, S. Plaszczynski, B. Rouill d'Orfeuil, M. Spinelli and M. Tristram, "Relieving tensions related to the lensing of the cosmic microwave background temperature power spectra," Astron. Astrophys. 597, A126 (2017) doi:10.1051/0004-6361/201527740 arXiv:1510.07600 [astro-ph.CO]].

[17] P. Motloch and W. Hu, "Lensinglike tensions in the Planck legacy release," Phys. Rev. D 101, no.8, 083515 (2020) doi:10.1103/PhysRevD.101.083515 arXiv:1912.06601 [astro-ph.CO]].

[18] G. Addison, Y. Huang, D. Watts, C. Bennett, M. Halpern, G. Hinshaw and J. Weiland, "Quantifying discordance in the 2015 Planck CMB spectrum," Astrophys. J. 818, no.2, 132 (2016) doi:10.3847/0004637X/818/2/132 arXiv:1511.00055 [astro-ph.CO]].

[19] W. Hadley, Curvature tension: evidence for a closed universe, arXiv:1908.09139 [astoph-CO]].

[20] E. Di Valentino, A. Melchiorri and J. Silk, "Planck evidence for a closed Universe and a possible crisis for cosmology," Nat. Astron. (2019) arXiv:1911.02087 [astroph.CO]].

[21] A. Ashtekar and P. Singh, "Loop quantum cosmology: A status report," Class. Quant. Grav. 28, 213001 (2011), arXiv:1108.0893 [gr-qc]].

[22] I. Agullo and P. Singh, In Loop Quantum Gravity: The first 30 years, edited by A. Ashtekar and J. Pullin, (World Scientific, Singapore, 2017), arXiv:1612.01236 [gr-qc]].

[23] I. Agullo, A. Ashtekar and W. Nelson, "A quantum gravity extension of the inflationary scenario," Phys. Rev. Lett. 109, 251301 (2012), arXiv:1209.1609 [gr-qc]].

[24] I. Agullo, A. Ashtekar and W. Nelson, "The preinflationary dynamics of loop quantum cosmology: Confronting quantum gravity with observations," Class. Quant. Grav. 30, 085014 (2013), arXiv:1302.0254 [gr$\mathrm{qc}]$.

[25] M. Fernandez-Mendez, G. A. Mena Marugan, and J. Olmedo, "Hybrid quantization of an inflationary universe," Phys. Rev. D 86, 024003 (2012), arXiv:1205.1917[gr-qc]]. 
[26] A. Barrau, T. Cailleteau, J. Grain, and J. Mielczarek, "Observational issues in loop quantum cosmology," Class. Quant. Grav. 31 (2014) 053001, [arXiv:1309.6896 [gr-qc]].

[27] A. Ashtekar and A. Barrau, "Loop quantum cosmology: From pre-inflationary dynamics to observations," Class. Quant. Grav. 32, 234001 (2015), arXiv:1504.07559 [gr$\mathrm{qc}]$.

[28] I. Agullo and N. A. Morris, "Detailed analysis of the predictions of loop quantum cosmology for the primordial power spectra," Phys. Rev. D 92, 124040 (2015), arXiv:1509.05693 [gr-qc]].

[29] I. Agullo, "Loop quantum cosmology, non-Gaussianity, and CMB power asymmetry," Phys. Rev. D 92, 064038 (2015) arXiv:1507.04703.

[30] A. Ashtekar and B. Gupt, "Quantum gravity in the sky: Interplay between fundamental theory and observations," Class. Quant. Grav. 34, 014002 (2017), arXiv:1608.04228 [gr-qc]].

[31] A. Ashtekar and B. Gupt, "Initial conditions for cosmological perturbations," Class. Quant. Grav. 34, 035004 (2017), arXiv:1610.09424 [gr-qc]].

[32] L. C. Gomar, G. A. Mena-Marugan, D. M. De Blas, and J. Olmedo, "Hybrid loop quantum cosmology and predictions for the cosmic microwave background," Phys. Rev. D96, 103528 (2017), arXiv:1702.06036 [gr-qc]].

[33] I. Agullo, B. Bolliet and V. Sreenath, "Non-Gaussianity in loop quantum cosmology," Phys. Rev. D97, 066021 (2018), arXiv:1712.08148 [gr-qc]].

[34] V. Sreenath, I. Agullo and B. Bolliet, "Computation of non-Gaussiantity in loop quantum cosmology," arXiv: 1904.01075 [gr-qc].

[35] I. Agullo and L. Parker, "Stimulated creation of quanta during inflation and the observable universe," Gen. Rel. Grav. 43, 2541 (2011) arXiv:1106.4240 [astro-ph.CO]]; I. Agullo and L. Parker, "Non-gaussianities and the Stim- ulated creation of quanta in the inflationary universe," Phys. Rev. D 83, 063526 (2011) arXiv:1010.5766 [astroph.CO]].

[36] J. Ganc and E. Komatsu, Scale-dependent bias of galaxies and mu-type distortion of the cosmic microwave background spectrum from single-field inflation with a modified initial state, Phys. Rev. D 86, 023518 (2012) [arXiv: 1204.4241 [astro-ph.CO]]

[37] R. Penrose, "The Road to Reality (section 28.8)," Alfred A Knopf, NY, 2004.

[38] A. Bhardwaj, E. J. Copeland, J. Louko, "Inflation in Loop Quantum Cosmology," Phys. Rev. D99, 063520 (2019) arXiv:1812.06841 [gr-qc]].

[39] A. A. Starobinsky, "A new type of isotropic cosmological models without singularity," Phys. Lett. 91B, 99 (1980) [Adv. Ser. Astrophys. Cosmol. 3, 130 (1987)]. doi:10.1016/0370-2693(80)90670-X

[40] I. Agullo, D. Kranas and V. Sreenath, Anomalies in the CMB from a cosmic bounce, [2005.01796 [astro-ph.CO]].

[41] A. Fialkov and A. Loeb, "Precise Measurement of the Reionization Optical Depth from The Global 21-cm Signal Accounting for Cosmic Heating," Astrophys. J. 821, no. 1, 59 (2016) doi:10.3847/0004-637X/821/1/59 arXiv:1601.03058 [astro-ph.CO]].

[42] T. Matsumura et al., "Mission design of LiteBIRD," J. Low. Temp. Phys. 176, 733 (2014) arXiv:1311.2847 [astro-ph.IM]].

[43] J. Delabrouille et. al. [CORE Collaboration] "Exploring cosmic origins with CORE: Survey requirements and mission design" JCAP, 2018, 04, 014 (2018).

[44] S. Hanany et al. [NASA PICO Collaboration], "PICO: Probe of Inflation and Cosmic Origins," arXiv:1902.10541 [astro-ph.IM].

[45] http://www.hpc.lsu.edu 